\title{
Intravitreal Voriconazole for the Treatment of Cryptococcus neoformans Endogenous Endophthalmitis
}

\author{
Asghar A. Haider, MD, MBA, ${ }^{1}$ J. R. Gallagher, MD, ${ }^{1}$ Jordan S. Johnson, MD, ${ }^{2}$ Joseph D. Benevento, MD² \\ ${ }^{1}$ Department of Ophthalmology, Louisiana State University Health Sciences Center, New Orleans, LA ${ }^{2}$ Department of Ophthalmology, \\ Ochsner Clinic Foundation, New Orleans, LA
}

Background: Cryptococcus neoformans is an encapsulated yeast that can cause fungemia and, in rare instances, lead to endogenous fungal endophthalmitis. No standard of care has been established to treat fungal endophthalmitis when systemic antifungal treatment fails to resolve the intraocular infection. Intravitreal voriconazole has been used for the treatment of fungal endophthalmitis caused by a broad range of fungal pathogens, and a limited number of reports have shown the efficacy of using intravitreal voriconazole for $C$ neoformans endophthalmitis. We report a case of endogenous fungal endophthalmitis caused by $C$ neoformans that was responsive to intravitreal voriconazole.

Case Report: A previously healthy 57-year-old male diagnosed with primary neuroendocrine lung tumor developed endogenous endophthalmitis from C neoformans. The endophthalmitis was resistant to intravenous amphotericin B treatment but was responsive to intravenous fluconazole in one eye and was apparently more responsive to intravitreal voriconazole in the other eye.

Conclusion: Intravitreal voriconazole should be considered for the treatment of cryptococcal endophthalmitis.

Keywords: Cryptococcus, Cryptococcus neoformans, endophthalmitis, eye infections-fungal, intravitreal injections, voriconazole

Address correspondence to Joseph D. Benevento, MD, Department of Ophthalmology, Ochsner Clinic Foundation, 1514 Jefferson Hwy., New Orleans, LA 70121. Tel: (504) 842-3000. Email: joseph.benevento@ochsner.org

\section{INTRODUCTION}

Cryptococcus neoformans is an encapsulated yeast commonly found in soil. ${ }^{1}$ Several different species can cause cryptococcal disease, and based on a 2015 phylogenetic analysis, 7 distinct species have been identified, with the most common pathogen being $C$ neoformans. ${ }^{2}$ Infection from $C$ neoformans usually presents as pneumonia and spreads hematogenously to other organs. Ocular involvement usually occurs after cryptococcal meningitis from hematogenous dissemination through the leptomeninges. Intraocular manifestation includes vitritis, choroiditis, exudative retinal detachment, neuroretinitis, and endophthalmitis. ${ }^{1,3}$ Cryptococcal endophthalmitis is an extremely rare form of fungal endophthalmitis and is typically treated with intravenous (IV) amphotericin B. ${ }^{4-6}$ Delayed diagnosis and lack of standardized treatment protocols make fungal endophthalmitis challenging to treat, and few cases of successful treatment of cryptococcal endophthalmitis have been reported. ${ }^{7}$

Patients with fungal endophthalmitis usually have multiple predisposing systemic conditions, including recent hospitalization, diabetes mellitus, liver disease, renal failure, cancer, indwelling lines, systemic surgery, organ transplantation, human immunodeficiency virus/acquired immunodeficiency syndrome, IV drug use, and immunosuppressive therapy, although cases of healthy immunocompetent patients have also been reported. . $^{3,8}$
When traditional systemic antifungal treatment is inadequate, alternative approaches such as newer classes of antifungal drugs, vitrectomy, and intravitreal injection have shown promise. ${ }^{9}$ We report a case of endogenous fungal endophthalmitis caused by $C$ neoformans that was responsive to intravitreal voriconazole.

\section{CASE REPORT}

A 57-year-old previously healthy male was found to have biopsy-proven primary right lung neuroendocrine tumor while being treated for pneumonia. During his malignancy evaluation, he developed altered mental status and experienced multiple cerebral embolic vascular accidents. No source of emboli was found with either transesophageal or transthoracic echocardiogram. The etiology was postulated to be the hypercoagulable state from the underlying malignancy. The plan was surgical removal of the tumor; however, during the patient's hospital stay, his clinical status declined, and he required ventilator support to maintain his oxygen saturation. He was transferred to our tertiary care center for further workup and treatment.

Magnetic resonance imaging of the brain was concerning for leptomeningeal inflammation and hydrocephalus. Lumbar puncture revealed cryptococcal antigen, and induction therapy with amphotericin B $5 \mathrm{mg} / \mathrm{kg}$ IV every 24 hours and flucytosine $25 \mathrm{mg} / \mathrm{kg}$ IV every 6 hours for 4 weeks was begun for the treatment of cryptococcal meningitis. The patient also 


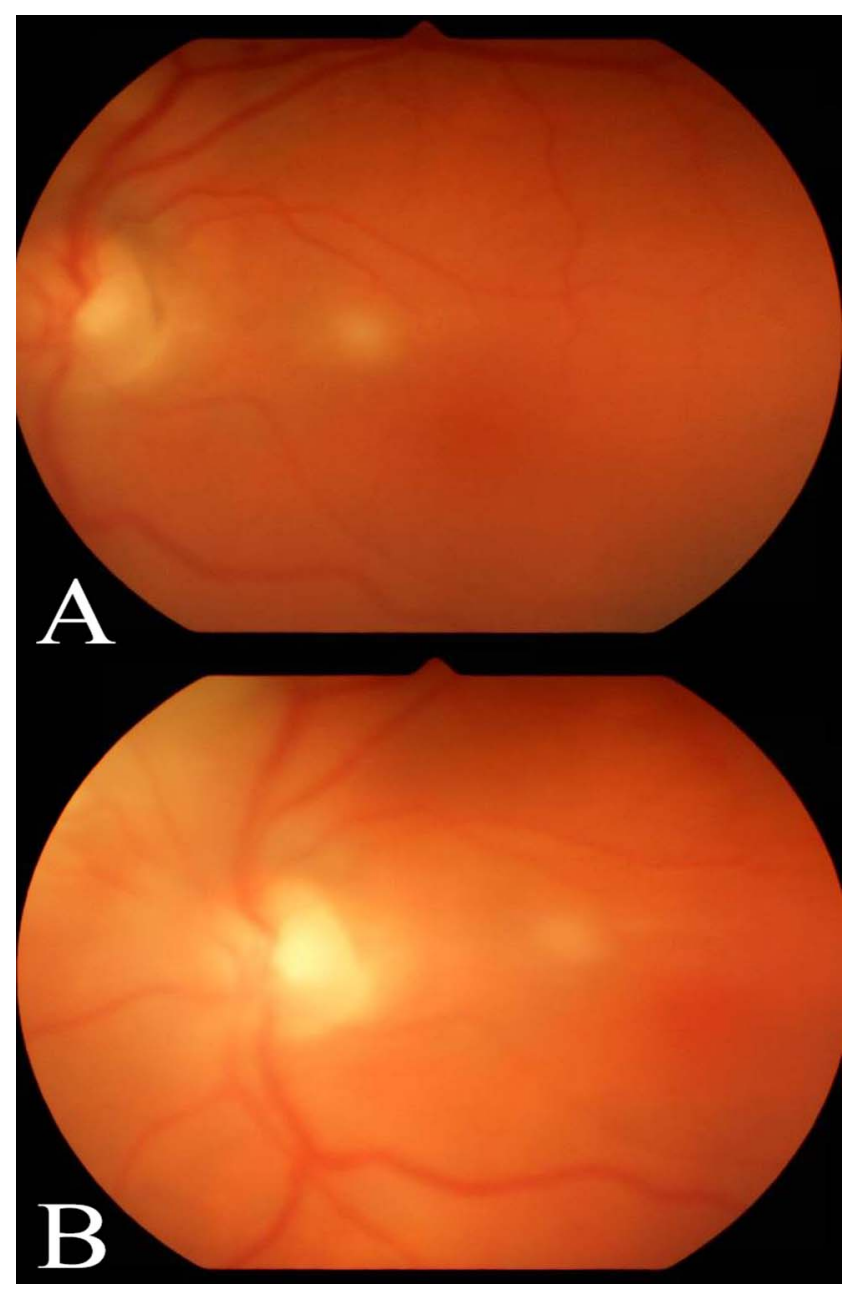

Figure 1. (A) Fundus photograph of the left eye from the initial dilated examination demonstrates a macular lesion with overlying vitritis. (B) Fundus photograph 4 weeks after initial evaluation shows regression of lesion.

received ampicillin $2 \mathrm{~g}$ IV every 4 hours, ceftriaxone $2 \mathrm{~g} \mathrm{IV}$ every 12 hours, and vancomycin $1,500 \mathrm{mg}$ once, followed by $1,000 \mathrm{mg}$ every 12 hours. An external ventricular drain was placed to monitor intracranial pressure (ICP). After multiple lumbar punctures with elevated opening pressures and concern for uncontrolled ICP, a ventriculoperitoneal shunt (VPS) was placed.

Ophthalmology was consulted approximately 1 month after the patient was transferred. Dilated fundus examination revealed bilateral multifocal, elevated, white chorioretinal infiltrates with vitritis. The left eye had a macular lesion (Figure $1 \mathrm{~A}$ ) and a peripapillary lesion that was more elevated and appeared to penetrate more into the vitreous than the other lesions (Figures 2A, 2B, and 2C). The right eye had a non-macula-threatening infiltrate (Figure $3 \mathrm{~A}$ ). At that time, the systemic amphotericin $B$ was changed to fluconazole $800 \mathrm{mg}$ IV every 12 hours.

Repeat dilated examination of the left eye showed progression of the peripapillary lesion (Figure 2D), prompting a vitreous tap and intravitreal injection of $0.1 \mathrm{~mL}$ voriconazole $(100 \mu \mathrm{g} / 0.1 \mathrm{~mL})$. The patient's vitreous cultures were negative, and serial examinations revealed initial stability followed by gradual improvement (Figures $2 \mathrm{E}$ and $2 \mathrm{~F}$ ). One week later, the intravitreal injection of voriconazole was repeated in the left eye because of the persistence of possible early retina breakthrough of the nasal infiltrate. The infiltrate in the right eye followed a nonprogressive course and thus was not treated with intravitreal injections. The infiltrates in the left eye showed regression, with early chorioretinal atrophic scarring around fading infiltrates (Figures 1B, 2E, and 2F). The lesion in the right eye (Figure $3 \mathrm{~B}$ ) followed the same course. Complete resolution was not documented because the patient transferred to an out-of-state specialty center.

\section{DISCUSSION}

$C$ neoformans causing endogenous endophthalmitis is rare, and the exact incidence is unknown. ${ }^{10}$ Candida species cause the majority of reported cases of fungal endophthalmitis, and most occur in immunocompromised hosts. $3,5,11,12$ The literature provides limited guidance for the treatment of fungal endophthalmitis caused by other species such as $C$ neoformans. Effective treatment is further challenged by the difficulty of early diagnosis and the ocular efficacy of commonly used systemic antifungals.

Our patient had several risk factors for developing endogenous fungal endophthalmitis, including recent hospitalization, cancer, respiratory disease, and presence of an IV line. ${ }^{3}$ Additionally, according to 2 recent (2018 and 2016) reports, our patient's VPS may have also predisposed him to cryptococcal infection. ${ }^{7,13}$ Of these 2 reported cases of VPS-associated Cryptococcus, 1 patient did not have ocular involvement, and the symptoms rapidly resolved with the removal of VPS and administration of oral flucytosine and IV amphotericin B. ${ }^{13}$ The patient in the case reporting fungal endophthalmitis associated with a VPS had a poor response to amphotericin B, but the lesion was stabilized with systemic fluconazole. ${ }^{7}$ These cases highlight the potential link between cryptococcal infection and VPS.

Our patient underwent an ophthalmic evaluation after he was noted to have ventilator-related conjunctival chemosis. Altered mental status and significant comorbidities prevented our patient from reporting changes in visual acuity or pain with eye movement, potentially delaying more timely ophthalmology consultation.

As stated previously, initial treatment for endogenous fungal endophthalmitis usually involves systemic amphotericin B. ${ }^{4}$ The many well-studied disadvantages of using systemic amphotericin B include questionable ocular penetration, poor side effect profile, and retinal toxicity. ${ }^{14}$ The lack of consensus for an alternative treatment usually means that other antifungals are only used when initial treatment is ineffective, possibly causing an unnecessary delay in optimal treatment.

Newer antifungals are available and have shown efficacy in the treatment of fungal endophthalmitis. ${ }^{4,5,15}$ Antifungal susceptibility studies have shown that an increasing number of clinical isolates are more susceptible to voriconazole and other antifungal agents compared to amphotericin B alone. ${ }^{6,16}$ Our patient received amphotericin B and flucytosine, which were sufficient to stabilize the lesions in the right eye. However, intravitreal voriconazole was used for the persistent lesions in the left eye. This clinical course supports the use of systemic antifungal therapy in fungal endophthalmitis, especially when the disease is bilateral, but 


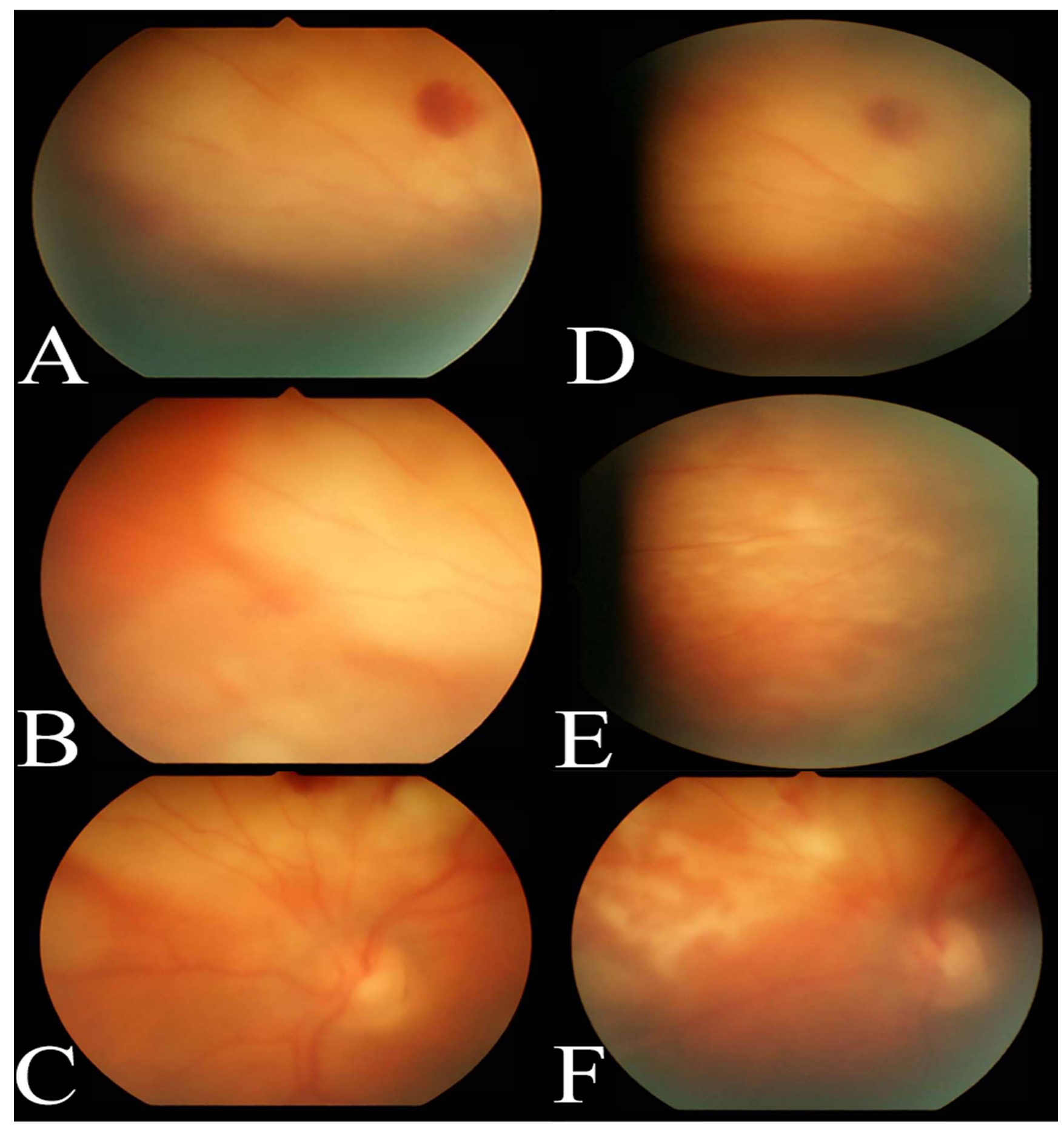

Figure 2. Fundus photographs of the left eye from the initial evaluation show a peripapillary lesion (A) with white chorioretinal infiltrates with vitritis (B and C). The peripapillary lesion showed worsening vitritis indicating progression at 1 week (D). Vitritis and chorioretinal infiltrates showed regression at the subsequent 2-week (E) and 4-week visits (F).

also highlights the importance of combination therapy and the use of intravitreal antifungal therapy for more aggressive disease. This strategy of combination therapy with multiple antifungal agents has been advocated by other authors for treatment of cryptococcosis. ${ }^{6,15}$

Of note, our patient did receive a long course of combination systemic antifungal medications, and this treatment may explain why no organism was isolated from the vitreous fluid biopsy and blood cultures. Treatment response assessment was based on his clinical examination that showed a significant improvement in vitreoretinal lesions after intravitreal injection in the left eye.

\section{CONCLUSION}

Intravitreal voriconazole as primary treatment or in combination with systemic antifungal medication should be 


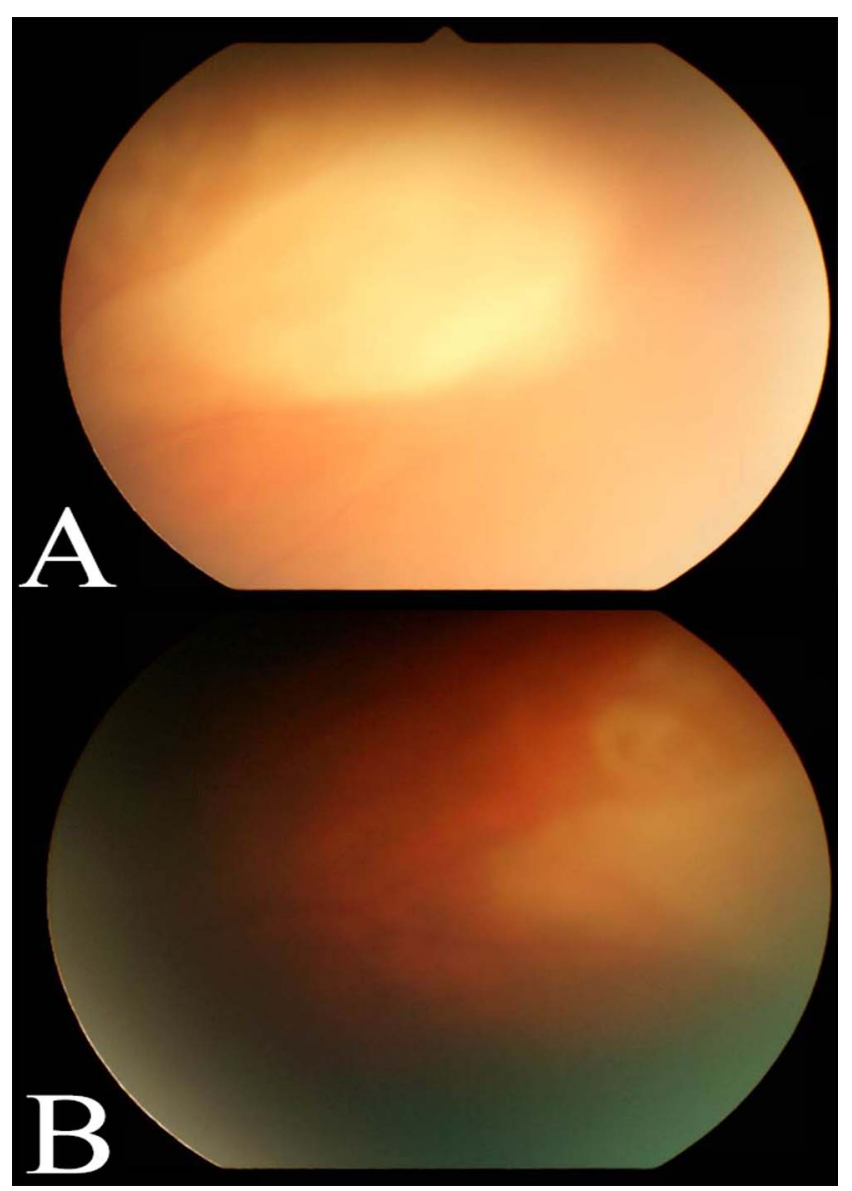

Figure 3. Fundus photograph of the right eye 1 week after treatment $(A)$ demonstrates a large chorioretinal infiltrate with overlying vitritis. The lesion responded well to treatment with a markedly regressed appearance at 2-week follow-up (B).

considered in patients with $C$ neoformans fungal endophthalmitis. Studies are needed to evaluate dosing, optimal combination therapy, and appropriate follow-up intervals, but given the rarity of cryptococcal fungal endophthalmitis, a multicenter collaboration would likely be necessary.

\section{ACKNOWLEDGMENTS}

The authors have no financial or proprietary interest in the subject matter of this article.

\section{REFERENCES}

1. Sheu SJ, Chen YC, Kuo NW, Wang JH, Chen CJ. Endogenous cryptococcal endophthalmitis. Ophthalmology. 1998 Feb; 105(2):377-381.

2. Hagen F, Khayhan K, Theelen B, et al. Recognition of seven species in the Cryptococcus gattii/Cryptococcus neoformans species complex. Fungal Genet Biol. 2015 May;78:16-48. doi: 10.1016/j.fgb.2015.02.009.

3. Lingappan A, Wykoff CC, Albini TA, et al. Endogenous fungal endophthalmitis: causative organisms, management strategies, and visual acuity outcomes. Am J Ophthalmol. 2012 Jan;153(1):162-166.e1. doi: 10.1016/j.ajo.2011.06.020.

4. Hariprasad SM, Mieler WF, Lin TK, Sponsel WE, Graybill JR. Voriconazole in the treatment of fungal eye infections: a review of current literature. Br J Ophthalmol. 2008 Jul;92(7):871-878. doi: 10.1136/bjo.2007.136515.

5. Durand ML. Bacterial and fungal endophthalmitis. Clin Microbiol Rev. 2017 Jul;30(3):597-613. doi: 10.1128/CMR.00113-16.

6. Reichert-Lima F, Busso-Lopes AF, Lyra L, et al. Evaluation of antifungal combination against Cryptococcus spp. Mycoses. 2016 Sep;59(9):585-593. doi: 10.1111/myc.12510.

7. Amphornphruet A, Silpa-Archa S, Preble JM, Foster CS. Endogenous cryptococcal endophthalmitis in immunocompetent host: case report and review of multimodal imaging findings and treatment. Ocul Immunol Inflamm. 2018;26(4):518-522. doi: 10.1080/09273948.2017.1298820.

8. Valluri S, Moorthy RS, Liggett PE, Rao NA. Endogenous Aspergillus endophthalmitis in an immunocompetent individual. Int Ophthalmol. 1993 Jun;17(3):131-135. doi: 10.1007/bf00942926.

9. Breit SM, Hariprasad SM, Mieler WF, Shah GK, Mills MD, Grand MG. Management of endogenous fungal endophthalmitis with voriconazole and caspofungin. Am J Ophthalmol. 2005 Jan;139(1):135-140. doi: 10.1016/j.ajo.2004.08.077.

10. Schiedler V, Scott IU, Flynn HW Jr, Davis JL, Benz MS, Miller D. Culture-proven endogenous endophthalmitis: clinical features and visual acuity outcomes. Am J Ophthalmol. 2004 Apr;137(4):725-731. doi: 10.1016/j.ajo.2003.11.013.

11. Essman TF, Flynn HW Jr, Smiddy WE, et al. Treatment outcomes in a 10-year study of endogenous fungal endophthalmitis. Ophthalmic Surg Lasers. 1997 Mar;28(3):185-194.

12. Binder MI, Chua J, Kaiser PK, Procop GW, Isada CM. Endogenous endophthalmitis: an 18-year review of culture-positive cases at a tertiary care center. Medicine (Baltimore). 2003 Mar;82(2):97-105. doi: 10.1097/00005792-200303000-00004.

13. Foong KS, Lee A, Vasquez G. Cryptococcal infection of the ventriculoperitoneal shunt in an immunocompetent patient. Am J Case Rep. 2016 Jan;17:31-34. doi: 10.12659/ajcr.896171.

14. O'Day DM, Head WS, Robinson RD, Stern WH, Freeman JM. Intraocular penetration of systemically administered antifungal agents. Curr Eye Res. 1985 Feb;4(2):131-134. doi: 10.3109/02713688508999978.

15. Silva RA, Sridhar J, Miller D, Wykoff CC, Flynn HW Jr. Exogenous fungal endophthalmitis: an analysis of isolates and susceptibilities to antifungal agents over a 20-year period (1990-2010). Am J Ophthalmol. 2015 Feb;159(2):257-264.e1. doi: 10.1016/j.ajo.2014.10.027.

16. Shen YC, Wang MY, Wang CY, et al. Clearance of intravitreal voriconazole. Invest Ophthalmol Vis Sci. 2007 May;48(5):2238-2241. doi: 10.1167/iovs.06-1362.

This article meets the Accreditation Council for Graduate Medical Education and the American Board of Medical Specialties Maintenance of Certification competencies for Patient Care and Medical Knowledge.

(C2020 by the author(s); licensee Ochsner Journal, Ochsner Clinic Foundation, New Orleans, LA. This article is an open (c) (i) access article distributed under the terms and conditions of the Creative Commons Attribution (CC BY) license (creativecommons.org/licenses/by/4.0/legalcode) that permits unrestricted use, distribution, and reproduction in any medium, provided the original author(s) and source are credited. 